\title{
Adverse Histological Features Alone Was Not Sufficient for Decision of Adjuvant Chemotherapy in T3NOMO colonic cancer: A Propensity Score Matching Study Based on Population Analysis
}

\section{Qinghua Wang}

Jinhua Hospital of Zhejiang University: Jinhua Municipal Central Hospital

Hongjuan Zheng

Jinhua Hospital of Zhejiang University: Jinhua Municipal Central Hospital

Xiaoxiao Chen

Jinhua Hospital of Zhejiang University: Jinhua Municipal Central Hospital

Xia Zhang

Jinhua Hospital of Zhejiang University: Jinhua Municipal Central Hospital

Xiayun Jin

Jinhua Hospital of Zhejiang University: Jinhua Municipal Central Hospital

Wanfen Tang

Jinhua Hospital of Zhejiang University: Jinhua Municipal Central Hospital

Xifeng Xu

Jinhua Hospital of Zhejiang University: Jinhua Municipal Central Hospital

Shishi Zhou

Jinhua Hospital of Zhejiang University: Jinhua Municipal Central Hospital

Jinlin Du

Jinhua Hospital of Zhejiang University: Jinhua Municipal Central Hospital

Jianfei Fu ( $\sim 11218276 @ z j u . e d u . c n)$

Affiliated Jinhua Hospital, Zhejiang University School of Medicine https://orcid.org/0000-0002-3036-1056

\section{Research article}

Keywords: Colonic cancer, Stage II, Adjuvant chemotherapy, Cancer specific survival (CSS), SEER, Propensity score matching (PSM)

Posted Date: January 19th, 2021

DOl: https://doi.org/10.21203/rs.3.rs-148512/v1

License: (c) (1) This work is licensed under a Creative Commons Attribution 4.0 International License. Read Full License 


\section{Abstract}

Purpose: Colorectal cancer (CRC) is one of the leading causes of cancer-related deaths worldwide. Approximately $25 \%$ of patients with AJCC stage II suffered recurrence after radical surgery, but the role of adjuvant chemotherapy in Stage II CRC is controversial. We aimed to evaluate the benefit from chemotherapy for T3NOMO patients with adverse histological features.

Methods: An analysis of the Surveillance, Epidemiology, and End Results (SEER) 18 databases was performed to identify patients underwent radical resection 20-80 years old with colonic carcinoma diagnosed during year 2004 to 2012. Poorly differentiated, undifferentiated, mucinous adenocarcinoma and signet ring cell carcinoma (SRCC) were defined as adverse histological features, and well or moderately differentiated adenocarcinoma as good histological features. Multivariate analyses were used to adjust the effects of other covariates. The survival benefit of adjuvant chemotherapy was demonstrated in the propensity score matching cohort.

Results: The adjuvant chemotherapy brought no survival benefit (good histological features HR, 1.076; 95\% $\mathrm{Cl}, 0.957-1.211 ; P=0.220$; adverse histological features $\mathrm{HR}, 0.995 ; 95 \% \mathrm{Cl}, 0.777-1.274 ; P=0.967)$; Neither mucinous adenocarcinoma nor signet ring cell carcinoma brought a significance on survival (mucinous adenocarcinoma HR, 0.699; $95 \% \mathrm{Cl}, 0.488-1.002 ; P=0.051$; signet ring cell carcinoma $\mathrm{HR}, 0.750 ; 95 \% \mathrm{Cl}, 0.386$ 1.459; $P=0.397$ ) compared to adenocarcinoma. Left-side colon was associated with a poorer survival in good histological features group (HR, 1.136; $95 \% \mathrm{Cl}, 1.034-1.248 ; P=0.008)$.

Conclusions: The adverse histological features don't influence on survival for patients with T3NOM0 staging, and adjuvant chemotherapy is invalid to improve the survival for these patients, indicating adverse histological features alone was not sufficient for decision of adjuvant chemotherapy in this situation rather than the recommendation of the guidelines.

\section{Introduction}

With its incidence currently increasing, colorectal cancer (CRC) is one of the most common cancer types worldwide contributing a major cause of cancer death, and $24-27 \%$ have stage II and $27-31 \%$ stage III disease[1, 2]. Although an benefit of adjuvant treatment with fluoropyrimidines is confirmed to improves the outcome of stage II/III cancer patients with radically resected colorectal cancer[3], controversy is common about the current clinical guidelines for stage II disease, especially when it refers to the benefit of the adjuvant chemotherapy[3]. Current clinical algorithms are unable to precisely predict which colorectal cancer patients would benefit from adjuvant chemotherapy. Adjuvant fluoropyrimidine-based chemotherapy is commonly administrated for colonic carcinoma in patients with stage III and to highly selective stage II with risks including T4 status, poorly differentiated histology, tumor perforation and insufficient examined lymph nodes[4-6], with expectation that high-risk patients could benefit from fluorouracil adjuvant chemotherapy, however, the survival benefit has not been confirmed[2]. Adjuvant chemotherapy in stage II colonic cancer is still a matter of debate and remains a challenge due to the limited survival benefit and considerable toxicity. Identification of prognostic and predictive biomarkers would contribute to the improvement of strategies to optimize selection of treatment options. A careful evaluation of precise benefit for the completely resected 
colonic cancer with stage II disease is of great significance, especially for patients staging as T3NOMO with so called "high risks".

We sought to facilitate a more practical approach based on predictive and prognostic factors, so as to prove the survival benefit of chemotherapy with the aim to minimizing its side effects in a large cohort of patients from the SEER database.

\section{Methods}

\section{Study population}

It is feasible to investigate the prognostic factors of target disease using the data from the SEER database, which is sponsored by the National Cancer Institution. In this study, we identified individuals diagnosed with microscopically confirmed adenocarcinoma in detail of the colon excluding rectum and rectosigmoid junction from 2004-2012.

The current SEER database, which is an authoritative source of information in the United States, currently collects and publishes cancer data from population-based cancer registries across the United States, approximately covering $28 \%$ of the United States population[7, 8]. The SEER is publicly available (http://seer.cancer.gov/), and the SEER Stat software (SEER Stat 8.3.6) was utilized to access the research data (Reference Number: 12296-Nov2018). To ensure an adequate follow-up, the patients diagnosed after 2012 were excluded. The clinicopathological variables including age and year at diagnosis, marital status, race, sex, histologic grade, tumor type, tumor site, adjuvant chemotherapy, survival time and cause of death were retrieved.

The inclusion criteria were as follows: (1) patients were diagnosed form 2004 to 2012; (2) the site code (ICD0-3) was limited to colon whose diagnosis were pathologically confirmed excluding rectum and rectosigmoid junction (C18.0, C18.2-C18.7); (3) histology code (ICD-0-3) was restricted to adenocarcinoma, mucinous adenocarcinoma and signet ring cell carcinoma $(8140 / 3,8480 / 3,8490 / 3)$; (4) patients whose TNM staging were limited to T3NOMO (staging based on 6th or 7th AJCC system according to the version of the day); and (5) patients who underwent radical resection (D2 or D3 lymphadenectomy with negative resection margins (R0) ) with exact number of resected regional lymph nodes for evaluation; (6) age of patients was limited to 20 to 80 (less than) years old; (7) patients with one primary tumors; (8) information on CSS and survival months was available. (Fig. 1).

Figure 1. The inclusion and exclusion criteria in seer.

\section{Variable Declaration}

Age was grouped as less than 70 years old $(<70)$ and more than 70 years old $(\geq 70)$. Race status was divided into black, white and others. Marital status was divided to married, single or divorced. There were three histology groups, adenocarcinoma, mucinous adenocarcinoma or signet ring cell carcinoma (SRCC). Poorly differentiated or undifferentiated adenocarcinoma, mucinous adenocarcinoma and signet ring cell carcinoma 
(SRCC) were all defined as adverse histological features, while well or moderately differentiated adenocarcinoma were defined as good histological features. Tumor site was grouped as right-side colon and left-side colon whose cut-off was splenic flexure. Regardless of the sequence, adjuvant chemotherapy was grouped as no or yes.

\section{Statistical Analyses}

Cox proportional hazards regression model was used to conduct the multivariate analysis with backward, stepwise elimination of variables. HRs were adjusted for lymph nodes examined, age ( $<70$ or not), race, sex, marital status, tumor histology, grade and tumor location. The demographic and clinical characteristics of the cohorts are demonstrated in Table 1. Besides the factors mentioned above, patients were well balanced for lymphovascular invasion and perineural invasion. 
Table 1

Descriptive Characteristics of 19834 Patients of Study Population within the Surveillance, Epidemiology, and End Results (SEER) Medicare-linked Database and 1774 Propensity Score-Matched Patients within the Adverse Histological Features Population of Colonic Carcinoma

\begin{tabular}{|c|c|c|c|c|c|c|}
\hline \multirow[t]{2}{*}{ Characteristics } & \multicolumn{3}{|c|}{ Entire cohort $(n=19834)$} & \multicolumn{3}{|c|}{$\begin{array}{l}\text { Propensity score-matched cohort }(n= \\
1774)\end{array}$} \\
\hline & $\mathrm{GH}, \mathrm{n}(\%)$ & $\mathrm{AH}, \mathrm{n}(\%)$ & $\begin{array}{l}\mathrm{P} \\
\text { Value }\end{array}$ & $\begin{array}{l}\text { Chemotherapy, } \\
\text { n (\%) }\end{array}$ & $\begin{array}{l}\text { Non- } \\
\text { chemotherapy, } \\
\text { n (\%) }\end{array}$ & $\begin{array}{l}\mathrm{P} \\
\text { Value }\end{array}$ \\
\hline & $\begin{array}{l}n=16626 \\
(84.00)\end{array}$ & $\begin{array}{l}N=3208 \\
(16.00)\end{array}$ & & $887(50.00)$ & $887(50.00)$ & \\
\hline Age & & & 0.000 & & & 0.951 \\
\hline$<70$ & 11464(68.95) & 2043(63.68) & & 722(81.40) & 723(81.51) & \\
\hline$\geq 70$ & $5162(31.05)$ & 1165(36.32) & & 165(18.60) & 164(18.49) & \\
\hline Gender & & & 0.000 & & & 0.505 \\
\hline Female & $7801(46.92)$ & 1724(53.74) & & 482(54.34) & $468(52.76)$ & \\
\hline Male & $8825(53.80)$ & $1484(46.26)$ & & $405(45.66)$ & $419(47.24)$ & \\
\hline Race & & & 0.000 & & & 0.877 \\
\hline White & 12796(76.96) & $2679(83.51)$ & & 737(83.09) & 731(82.41) & \\
\hline Black & 2223(13.37) & 282(8.79) & & $73(8.23)$ & $79(8.91)$ & \\
\hline Other & 1607(9.67) & 247(7.70) & & $77(8.68)$ & $77(8.68)$ & \\
\hline Marital status & & & 0.002 & & & 0.981 \\
\hline Married & 10102(60.76) & 1896(59.10) & & $571(64.37)$ & $569(64.15)$ & \\
\hline Single & $2700(16.24)$ & 484(15.09) & & 140(15.78) & 143(16.12) & \\
\hline Divorce & $3824(23.00)$ & $828(25.81)$ & & 176(19.84) & 175(19.73) & \\
\hline Histology & & & 0.000 & & & 0.831 \\
\hline Adenocarcinoma & 16626(100.00) & $2760(86.03)$ & & 782(88.16) & 777(87.60) & \\
\hline $\begin{array}{l}\text { Mucinous } \\
\text { adenocarcinoma }\end{array}$ & $0(0.00)$ & $354(11.03)$ & & $84(9.47)$ & $85(9.58)$ & \\
\hline SRCC & $0(0.00)$ & $94(2.93)$ & & $21(2.37)$ & $25(2.82)$ & \\
\hline Grade & & & 0.000 & & & 0.148 \\
\hline
\end{tabular}

GH: Good histological features

AH: Adverse histological features

SRCC: signet ring cell carcinoma 


\begin{tabular}{|c|c|c|c|c|c|c|}
\hline \multirow[t]{2}{*}{ Characteristics } & \multicolumn{3}{|c|}{ Entire cohort $(n=19834)$} & \multicolumn{3}{|c|}{$\begin{array}{l}\text { Propensity score-matched cohort }(n= \\
\text { 1774) }\end{array}$} \\
\hline & $\mathrm{GH}, \mathrm{n}(\%)$ & $\mathrm{AH}, \mathrm{n}(\%)$ & $\begin{array}{l}\mathrm{P} \\
\text { Value }\end{array}$ & $\begin{array}{l}\text { Chemotherapy, } \\
\text { n (\%) }\end{array}$ & $\begin{array}{l}\text { Non- } \\
\text { chemotherapy, } \\
\text { n (\%) }\end{array}$ & $\begin{array}{l}\mathrm{P} \\
\text { Value }\end{array}$ \\
\hline Well & $1330(8.00)$ & $0(0.00)$ & & $0(0.00)$ & $0(0.00)$ & \\
\hline Moderate & $15296(92.00)$ & $0(0.00)$ & & $0(0.00)$ & $0(0.00)$ & \\
\hline Poor & $0(0.00)$ & 2912(90.77) & & $820(92.45)$ & 803(90.53) & \\
\hline Undifferentiated & $0(0.00)$ & 296(9.23) & & $67(7.55)$ & $84(9.47)$ & \\
\hline Tumor site & & & 0.000 & & & 0.810 \\
\hline Right side colon & $8081(48.60)$ & $2145(66.86)$ & & $521(58.74)$ & $516(58.17)$ & \\
\hline Left side colon & $8545(51.40)$ & 1063(33.14) & & $366(41.26)$ & $371(41.83)$ & \\
\hline Tumor deposit & & & & & & 0.950 \\
\hline Negative & $7067(42.50)$ & 962(29.99) & 0.000 & $329(37.10)$ & $327(36,87)$ & \\
\hline Positive & $482(2.90)$ & $129(4.02)$ & & $80(9.02)$ & $81(9.13)$ & \\
\hline Unknown & $9077(54.60)$ & $2117(65.99)$ & & $478(53.88)$ & $479((54.00)$ & \\
\hline $\begin{array}{l}\text { Peritoneum } \\
\text { invasion }\end{array}$ & & & 0.000 & & & 0.894 \\
\hline Negative & 7029(42.28) & $1284(40.02)$ & & $350(39.46)$ & 354(39.91) & \\
\hline Positive & $519(3.12)$ & $141(4.40)$ & & $87(9.81)$ & $85(9.58)$ & \\
\hline Unknown & $9078(54.60)$ & $1783(55.58)$ & & $450(50.73)$ & $448(50.51)$ & \\
\hline Chemotherapy & & & 0.000 & & & - \\
\hline Yes & $3653(21.97)$ & $887(27.65)$ & & - & - & \\
\hline No & 12973(78.03) & $2321(72.35)$ & & - & - & \\
\hline \multicolumn{7}{|c|}{ GH: Good histological features } \\
\hline \multicolumn{7}{|c|}{ AH: Adverse histological features } \\
\hline SRCC: signet ring & ell carcinoma & & & & & \\
\hline
\end{tabular}

Kaplan-Meier method was used to calculate the survival curves and to plot the survival curves. Differences of survival was compared by the log rank test. The 5-year cancer specific survival (CSS) was the primary endpoint in this study, and it was defined from the diagnosis to the date of cancer death. Death attributed to other causes was defined as a censored observation. Univariate and multivariate analyses were conducted respectively using Cox proportional hazards regression models to identify the factors which would influence the CSS independently either in population with good histological features or in population with adverse 
histological features. A method of propensity score matching (PSM) was utilized to balance the baseline characteristics of the population with adverse histological features between groups separated by whether administrated by chemotherapy or not. The propensity score was calculated by logistic regression including covariates of age ( $<70$ or not), race, sex, marital status, tumor histology, grade, tumor location, lymphovascular invasion and perineural invasion. The PSM cohort was prepared to validate the impact of chemotherapy on CSS in this population with adverse histological features. All statistical analyses were performed using R3.6.2 software (http://www.r-project.org/) or STATA/SE 15.0 software (StataCorp LP, College Station, TX, USA), and a two-sided value of $\mathrm{P}<0.05$ was considered statistically significant.

\section{Results}

Totally, 19834 eligible patients were enrolled in this study, and $16626(84.00 \%)$ were good histological features patients while the other $3208(16.00 \%)$ were adverse histological features patients. The median follow-up was 72.0 months (range 1.0-143.0 months), with a median age of 65.0 years (range 20.079.0 years). Table 1 presented the detailed clinicopathologic characteristics between the two groups.

Patients with adverse histological features were more likely to appear in women rather than men $(53.74 \%$ in women compared with $46.26 \%$ in men, $P<0.01$ ). Right side colon was a more frequent location lying the adverse histological features patients than left side colon $(66.86 \%$ compared with $33.14 \%, P<0.01)$. In reality, $22.89 \%$ of patients staging as T3NOMO (AJCC stage II) were sequential deal with adjuvant chemotherapy, which was more likely to be administrated in patients of adverse histological features histology $(27.65 \%$ in adverse histological features compared to $21.97 \%$ in good histological features, $P<0.01$ ). No significant difference was showed in the characteristics of 1774 PSM patients (Table 1).

Table 2 demonstrated the results of the univariate and multivariate analysis done to analyze predictors of OS of patients in the study population with good histological features. For patients with good histological features, the increasing number of lymph nodes resected for examination brought a significant survival benefit (hazard ratio [HR], 0.972; 95\% confidence interval [Cl], 0.966-0.977; $P=0.000$ ), while the increasing age appeared to significantly influence the survival $(\mathrm{HR}, 1.020 ; 95 \% \mathrm{Cl}, 1.013-1.027 ; P=0.000)$. Opposite to the knowledge well accepted in metastatic CRCs, left side colon appeared to be associated with a significantly negative influence on survival $(\mathrm{HR}, 1.136 ; 95 \% \mathrm{Cl}, 1.034-1.248 ; P=0.008)$. The adjuvant chemotherapy brought no benefit on survival $(\mathrm{HR}, 1.076 ; 95 \% \mathrm{Cl}, 0.957-1.211 ; P=0.220)$. 
Table 2

Univariate and Multivariate Analysis of Predictors of OS of Patients in the Study Population with Good Histological Features within the Surveillance, Epidemiology, and End Results (SEER) Medicare-linked database

\begin{tabular}{|c|c|c|c|c|c|c|}
\hline \multirow[t]{2}{*}{ Risk Factors } & \multicolumn{3}{|c|}{ Univariate analysis a } & \multicolumn{3}{|c|}{ Multivariate analysis ${ }^{\mathrm{b}}$} \\
\hline & HR & $\mathrm{HR}(95 \% \mathrm{Cl})$ & P Value & HR & HR (95\%Cl) & P Value \\
\hline LN examined & 0.966 & $0.961-0.971$ & 0.000 & 0.972 & $0.966-0.977$ & 0.000 \\
\hline Age(years) & 1.026 & $1.022-1.031$ & 0.000 & 1.020 & $1.013-1.027$ & 0.000 \\
\hline \multicolumn{7}{|l|}{ Age70(years) } \\
\hline Q70 & 1 & 1 & & 1 & 1 & \\
\hline$\geq 70$ & 1.664 & $1.520-1.822$ & 0.000 & 1.221 & $1.061-1.405$ & 0.005 \\
\hline \multicolumn{7}{|l|}{ Gender } \\
\hline Female & 1 & 1 & & 1 & 1 & \\
\hline Male & 1.154 & $1.055-1.262$ & 0.002 & 1.261 & $1.149-1.383$ & 0.000 \\
\hline \multicolumn{7}{|l|}{ Race } \\
\hline White & 1 & 1 & & 1 & 1 & \\
\hline Black & 1.339 & $1.189-1.509$ & 0.000 & 1.356 & $1.201-1.532$ & 0.000 \\
\hline Other & 0.910 & $0.776-1.067$ & 0.247 & 0.929 & $0.791-1.090$ & 0.367 \\
\hline \multicolumn{7}{|l|}{ Marital status } \\
\hline Married & 1 & 1 & & 1 & 1 & \\
\hline Single & 1.488 & $1.322-1.674$ & 0.000 & 1.593 & $1.412-1.798$ & 0.000 \\
\hline Divorced & 1.497 & $1.349-1.661$ & 0.000 & 1.408 & $1.262-1.569$ & 0.000 \\
\hline \multicolumn{7}{|l|}{ Grade } \\
\hline Well differentiated & 1 & 1 & & 1 & 1 & \\
\hline Moderately differentiated & 1.175 & $0.987-1.397$ & 0.069 & 1.183 & $0.994-1.408$ & 0.058 \\
\hline \multicolumn{7}{|l|}{ Tumor site } \\
\hline Right side colon & 1 & 1 & & 1 & 1 & \\
\hline Left side colon & 1.194 & $1.092-1.305$ & 0.000 & 1.136 & $1.034-1.248$ & 0.008 \\
\hline
\end{tabular}

a univariate and multivariate analyses were conducted using Cox regression model;

HR, hazard ratio;

LN examined: lymph nodes examined 


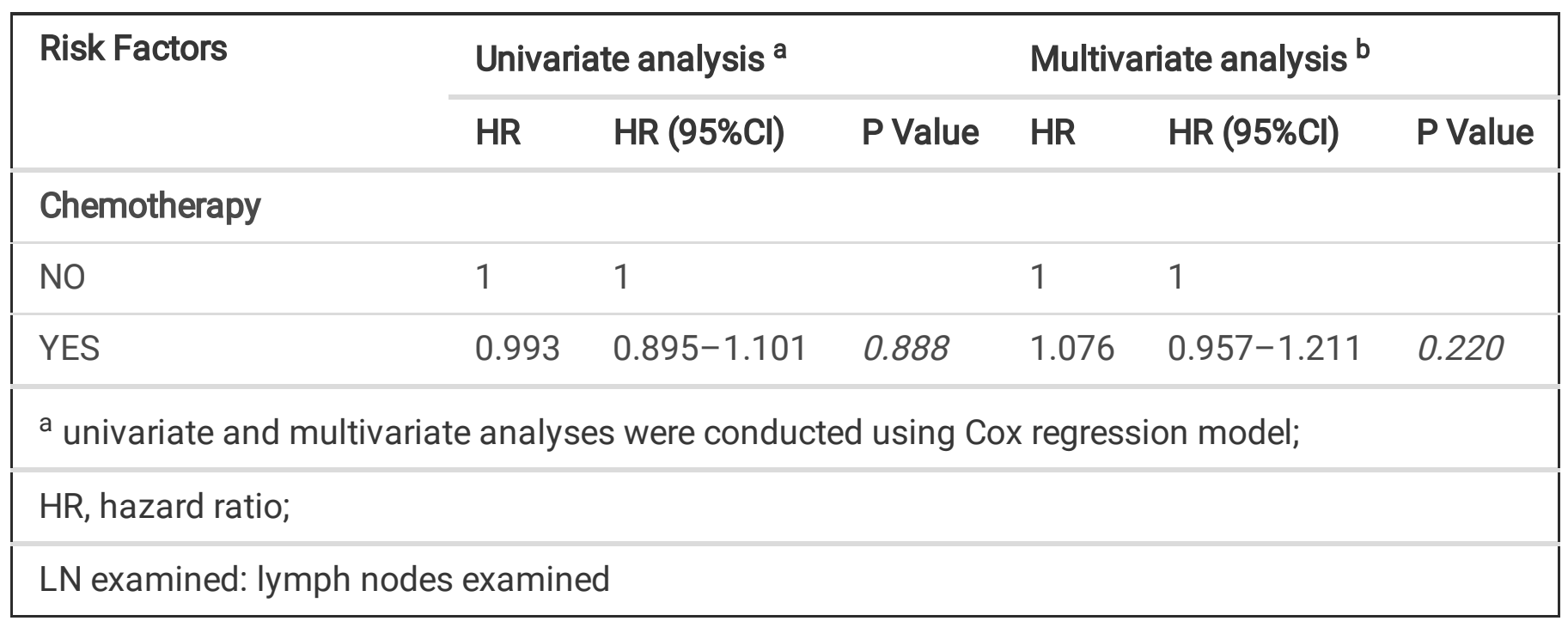

Table 3 showed the results of the univariate analysis and multivariate analysis done to analyze predictors of OS of patients in the study population with adverse histological features. For patients with adverse histological features, the increasing number of lymph nodes resected for examination still brought a significant survival benefit ( $\mathrm{HR}, 0.958 ; 95 \% \mathrm{Cl}, 0.947-0.970 ; P=0.000)$, while the increasing age appeared not to influence the survival ( $\mathrm{HR}, 1.012 ; 95 \% \mathrm{Cl}, 0.997-1.028 ; P=0.117)$. Compared to adenocarcinoma, neither mucinous adenocarcinoma nor signet ring cell carcinoma brought a significant influence on survival (mucinous adenocarcinoma $\mathrm{HR}, 0.699 ; 95 \% \mathrm{Cl}, 0.488-1.002 ; P=0.051$; signet ring cell carcinoma $\mathrm{HR}, 0.750$; 95\% Cl, 0.386-1.459; $P=0.397)$. Tumor site did not influence the survival ( $\mathrm{HR}, 1.007 ; 95 \% \mathrm{Cl}, 0.806-1.257 ; P$ $=0.953)$, and so did the adjuvant chemotherapy $(\mathrm{HR}, 0.995 ; 95 \% \mathrm{Cl}, 0.777-1.274 ; P=0.967)$. 
Table 3

Univariate and Multivariate Analysis of Predictors of OS In the Study Population with Adverse Histological Features within the Surveillance, Epidemiology, and End Results (SEER) Medicare-linked database

\begin{tabular}{|c|c|c|c|c|c|c|}
\hline \multirow[t]{2}{*}{ Risk Factors } & \multicolumn{3}{|c|}{ Univariate analysis ${ }^{a}$} & \multicolumn{3}{|c|}{ Multivariate analysis ${ }^{b}$} \\
\hline & HR & $\mathrm{HR}(95 \% \mathrm{Cl})$ & P Value & HR & $\mathrm{HR}(95 \% \mathrm{Cl})$ & P Value \\
\hline LN examined & 0.951 & $0.940-0.963$ & 0.000 & 0.958 & $0.947-0.970$ & 0.000 \\
\hline Age(years) & 1.026 & $1.016-1.036$ & 0.000 & 1.012 & $0.997-1.028$ & 0.117 \\
\hline Age70(years) & & & & & - & \\
\hline \$70 & 1 & 1 & & & - & \\
\hline$\geq 70$ & 1.784 & $1.463-2.176$ & 0.000 & 1.488 & $1.087-2.036$ & 0.013 \\
\hline Gender & & - & & & - & \\
\hline Female & & - & & & - & \\
\hline Male & 1.458 & $1.195-1.779$ & 0.000 & 1.630 & $1.322-2.010$ & 0.000 \\
\hline Race & & - & & & - & \\
\hline White & & - & & & - & \\
\hline Black & 1.670 & $1.245-2.239$ & 0.001 & 1.739 & $1.286-2.351$ & 0.000 \\
\hline Other & 0.793 & $0.523-1.201$ & 0.273 & 0.802 & $0.528-1.219$ & 0.302 \\
\hline Marital status & & - & & & - & \\
\hline Married & & - & & & - & \\
\hline Single & 1.321 & $1.000-1.744$ & 0.050 & 1.410 & $1.058-1.878$ & 0.019 \\
\hline Divorced & 1.357 & $1.081-1.703$ & 0.009 & 1.306 & $1.027-1.661$ & 0.029 \\
\hline Histology & & - & & & - & \\
\hline Adenocarcinoma & & - & & & - & \\
\hline Mucinous adenocarcinoma & 0.712 & $0.498-1.017$ & 0.062 & 0.699 & $0.488-1.002$ & 0.051 \\
\hline SRCC & 0.749 & $0.386-1.451$ & 0.392 & 0.750 & $0.386-1.459$ & 0.397 \\
\hline Grade & & - & & & - & \\
\hline
\end{tabular}

a univariate and multivariate analyses were conducted using Cox regression model;

HR, hazard ratio;

LN examined: lymph nodes examined

SRCC: signet ring cell carcinoma 


\begin{tabular}{|c|c|c|c|c|c|c|}
\hline \multirow[t]{2}{*}{ Risk Factors } & \multicolumn{2}{|c|}{ Univariate analysis $^{a}$} & \multicolumn{4}{|c|}{ Multivariate analysis ${ }^{b}$} \\
\hline & HR & HR $(95 \% \mathrm{Cl})$ & P Value & HR & HR $(95 \% \mathrm{Cl})$ & P Value \\
\hline Poor & & - & & & - & \\
\hline undifferentiated & 0.745 & $0.496-1.117$ & 0.154 & 0.819 & $0.545-1.230$ & 0.336 \\
\hline Tumor site & & - & & & - & \\
\hline Right side colon & & - & & & - & \\
\hline Left side colon & 1.194 & $0.973-1.465$ & 0.090 & 1.007 & $0.806-1.257$ & 0.953 \\
\hline Chemotherapy & & - & & & - & \\
\hline NO & & - & & & - & \\
\hline YES & 0.930 & $0.747-1.159$ & 0.520 & 0.995 & $0.777-1.274$ & 0.967 \\
\hline \multicolumn{7}{|c|}{ a univariate and multivariate analyses were conducted using Cox regression model; } \\
\hline \multicolumn{7}{|l|}{ HR, hazard ratio; } \\
\hline \multicolumn{7}{|c|}{ LN examined: lymph nodes examined } \\
\hline SRCC: signet ring & & & & & & \\
\hline
\end{tabular}

Figure 2 Survival analysis of colonic cancer patients with different features. A The cancer-specific survival (CSS) between good histological features (GD) and adverse histological features (BD) patients with T3N0M0 staging. B The CSS between right side and left side colon patients with T3NOMO staging. C The CSS between right side and left side colon patients of good histological features with T3N0M0 staging. D The CSS between right side and left side colon patients of adverse histological features with T3N0M0 staging. $P$ value from the log rank test.

Figure 3 Survival analysis of adjuvant chemotherapy in colonic cancer patients with T3NOMO staging. A The cancer-specific survival (CSS) between patients of good histological features with T3N0M0 staging with and without chemotherapy. B The CSS between patients of adverse histological features with T3N0M0 staging with and without chemotherapy. C The CSS between patients of adverse histological features with T3NOMO staging with chemotherapy and without chemotherapy in PSM cohort. P value from the log rank test.

\section{Discussion}

In the present study, we performed a propensity score matching study based on population analysis to explore the survival benefit of chemotherapy on stage II CRC and described three major findings: First, the adverse histological features of colonic cancer did not influence the survival at all for patients with T3N0M0 staging. Second, adjuvant chemotherapy seemed to be invalid to improve the survival of patients of adverse histological features with T3N0M0 staging, so it may be reasonable to subtract chemotherapy due to the considerable toxicity; Third, there has been a contradictory behavior of the tumor location as left side colonic 
cancer appeared to be associated with a poorer survival in population with good histological features whereas it's well accepted that right side colonic cancer represents a poorer survival, and the inflection point may lie in patients of adverse histological features with T3N0M0 staging just as illustrated in Fig. 2. Females remain at a lower risk for advanced stage CRC across all age groups, and probably benefit from protective effect against colon cancer of estrogen pathway[9] and microsatellite instability arising in females[10].

The therapy of rectal cancer is routinely referred to that of colonic cancer, however, these two diseases show their respective situation frequently, such as different lymphatic drainage pathway, molecular subtypes and application of radiotherapy, so only colonic cancer were included in the current study to minimize the bias. In this study, we also adopted the PSM to eliminate the difference of baseline between patients of adverse histological features with and without chemotherapy. PSM is a technique to improve the comparability between groups, as shown by the well-balanced demographics and clinicopathological characteristics in Table 1. Both the lymphovascular invasion and perineural invasion are recognized as adverse factors responsible for poor prognosis, so these two histological features were well balanced by PSM method to invalidate the confusion, despite of the actuality that the specific data was only on access from SEER database since year 2010.

The common adenocarcinoma (AC) accounts for the majority of colonic cancer cases, left 10-15\% patients with mucinous adenocarcinoma (MC) and approximately $1 \%$ of CRC patients with SRCC[11]. Although SRCC represents a rare condition, it is associated with a poor prognosis compared with AC[11, 12]. Five-year relative survival rate of SRCC in stage II colonic cancer patients was $67.9 \%$ compared with $77.0 \%$ of AC; while in stage II rectal cancer patients five-year relative survival rate of SRCC was $27.4 \%$ compared with $67.1 \%$ of $A C[11]$, indicating a significant influence of tumor location on survival, which was similar with our findings that the left side colonic cancer appeared to be associated with a poorer survival in population with good histological features opposite to the well accepted fact that right side colonic cancer represents a poorer survival in metastatic colorectal cancer. In concordance with SRCC, mucinous colorectal adenocarcinomas usually have poorer responses to chemotherapies[13], resulting in a poor prognosis in advanced disease[14]. Either MC or SRCC patients more often develop metastatic disease, and are more likely to develop peritoneal metastases and metastasizes via the lymphatic route, whereas AC metastasizes primarily to the liver[12], which may partially explains the poor prognosis of MC and SRCC. The standard treatments for colorectal adenocarcinoma are recommended to MC and SRCC patients since no clinical guidelines have been developed specifically for these groups of patients[13]. Due to the low frequency, SRCC and MC has not been well-studied in stage II colonic cancer patients and reports in the literature are uncommon. In the present study, poorly differentiated histology, which was routinely considered as an adverse prognostic factor, was integrated as adverse histological features with MC and SRCC together to illustrate the benefit of adjuvant chemotherapy in stage II colonic cancer excluding T4. In our study population, $22.89 \%$ of patients staging as T3NOMO were sequential deal with adjuvant chemotherapy, which was more likely to be administrated in patients of adverse histological features histology (27.65\% in adverse histological features compared to $21.97 \%$ in good histological features, $P<0.01)$. So, this kind of adverse histological features was accepted for clinicians to evaluate the necessity of chemotherapy. However, it is denied to administrate adjuvant chemotherapy in the current study for patients of adverse histological features, which is regarded currently as 
one of the high risks, implying the adverse histological features alone is not sufficient for the decision of adjuvant chemotherapy in T3NOMO.

Despite of the fact that inspiring therapeutic improvements have improved the survival rate in the last decade by an average of $3 \%$ every year $[15,16], C C$ serves as the 3rd cause of cancer deaths both in the world, no matter men or women[9], with almost $25 \%$ of patients with stage II disease suffering recurrence after radical surgery $[17,18]$. Surgery is the main therapeutic modality for CRC and adjuvant chemotherapy is the widely accepted pattern of administration with promising efficacy for stage III CC, on the consensus that the pathologic stage is the most helpful prognostic factor for the colorectal cancer[3,4]. Regardless of the recommendation of the NCCN guideline, the adjuvant chemotherapy benefit for stage II CRC is still controversial[19], and it's believed that only a few CRC patients with stage II would benefit from adjuvant therapy[17, 20]. Several stage-specific factors in stage II CRCs, including T4 lesions, positive margins, poorly differentiated histology, inadequately sampled lymph nodes and et. al[21], which are considered as high-risk stage II CRCs, provide certain prognostic value[22]. The 5-year disease-free survival could be more than $90 \%$ in patients without any risk factor, compared to $84.8 \%$ in patients with one or more risk factors[23]. So far, adjuvant chemotherapy is administrated for high-risk stage II CRC or later stage CRC since 2004[6], but there are insufficient data based on scientific evidence to confirm the efficacy of the biomarkers to select the appropriate candidate patients $[3,5]$ except that pathologic T4 stage was a significant factor predicting poor DFS[24], and only a trend for benefit from chemotherapy in the stage II was seen, failing to reach a significance[17]. Neither in the IMPACT trial nor the NSABP trial, improvement from adjuvant chemotherapy in survival of stage II CRC was seen[1], so the precise decision of whether a CC patient with Stage II disease should receive adjuvant chemotherapy is essential to improve the prognosis for every individual[19]. We came to the conclusion that adjuvant chemotherapy may be invalid to improve the survival even in patients of adverse histological features with T3NOMO staging, so it may be reasonable to subtract chemotherapy for patients of this situation in case of the presence of adverse histological features alone, and more precise indicators are essential for the selection of advantage cohort.

Nowadays, several researches have been carried out to identify the sensitive CRC patients with AJCC stage II for chemotherapy. Tumoral heterogeneity including KRAS[17, 25], BRAF, PTEN or microsatellite instability status[26], as well as tumor metabolism, such as NKX6.1 methylation[20], MMP7[27] and AQP1 [28], has been established on cancer response to targeted therapy or chemotherapy in metastatic CRCs[9], while it is of no uncertain use for stage II CRCs. Her-2 status should be a predictive factor, especially for patients with pMMR status, because Her-2 positive patients can benefit from 5-fluorouracial based adjuvant[29], while low expression of the PD-L1 gene was associated with benefit from adjuvant chemotherapy[30]. Despite of the efforts, there are now insufficient or reliable indicators to identify the proper patients suitable for adjuvant chemotherapy in stage II colonic cancer unfortunately, and the personalized intertumoral and genomic biomarkers would be promising in the future instead of clinicopathological indicators.

The current study had some limitations: First, as a retrospective analysis, it always carried the risk of various biases. Second, due to the carefully chosen criteria, only 887 colonic cancer patients of adverse histological features with T3NOMO staging who were administrated with adjuvant chemotherapy were enrolled in this study, which would influence the degree of power more or less. Third, the current SEER database is deficient 
in providing the entire picture of the registered patients such as genetic markers and disease-free survival (DFS), making it impossible to survey the influence of adverse histological features and other genomic biomarkers on DFS or CSS. So, more chemotherapeutic regimens are necessary to increase survival in this stage II CRC subset of patients and more valid genomic biomarkers are essential to come up with appropriate selection criteria.

The strengths of our study include: The current study minimized potential biases and had a higher degree of power with the use of a large-scale sample and PSM method. The segmentation of the high risks of the stage II CRCs, and then the only concern on adverse histological features was another merit. The SEER database allows researchers to accurately identify patients with good or adverse histological features based on histology and grade clearly, thus avoiding confusion of differentiation, and we colligate histology and grade of tumors in a new way intend to make the results more convincing. Still, the potential biases of surgery were avoided by super-selective cohort with the limit of radical resection.

\section{Conclusion}

According to the findings of our analysis of the SEER database, the adverse histological features does not influence on survival for patients with T3NOMO staging, and adjuvant chemotherapy is invalid to improve the survival of these patients, so adverse histological features alone was not sufficient for decision of adjuvant chemotherapy in this situation rather than the recommendation of the guidelines.

\section{Declarations}

\section{Acknowledgements}

The authors appreciate the efforts of the Surveillance, Epidemiology, and End Results (SEER) Program tumor registries in the creation of the SEER database and the Data Management team who generously spared their time for the accomplishment and fulfillment of this project.

\section{Ethics approval and consent to participate}

The study was approved by the institutional review board and ethics committee of Jinhua Central Hospital. All individual records were anonymous and de-identified prior to analysis.

\section{Competing Interests}

The authors have declared that no competing interest exists.

\section{Funding}

The study was partially supported by a grant from key program of the Jinhua Municipal Science \& Technology Bureau (grant number 2018-3-001d).

This study was supported by a grant from key program of scientific research of Jinhua Central Hospital (grant number JY2016-1-02). 
The study was partially supported by the Medical Science \& Technology Plan Project of Zhejiang Province (grant number LGF18H160029) and General program of Zhejiang Natural Science Foundation (grant number LY19H160020).

The study was partially supported by a grant from key program of the Jinhua Municipal Science \& Technology Bureau (grant number 2019-3-013).

\section{References}

1. Arakawa K, Kawai K, Tanaka T, et al. Prognostic impact of interhospital variation in adjuvant chemotherapy for patients with Stage II/III colorectal cancer: a nationwide study. Colorectal disease: the official journal of the Association of Coloproctology of Great Britain Ireland. 2018;20:0162-072.

2. Ishiguro $M$, Ueno $H$, Kanemitsu $Y$, et al. Current clinical practice of adjuvant chemotherapy for patients with 'high-risk' Stage II colorectal cancer in Japan: a questionnaire survey in the JCOG Study Group. Jpn J Clin Oncol. 2018;48:1109-12.

3. Cecchin E, Perrone G, Nobili S, et al. MTHFR-1298 A > C ( $r$ 1801131) is a predictor of survival in two cohorts of stage II/III colorectal cancer patients treated with adjuvant fluoropyrimidine chemotherapy with or without oxaliplatin. The pharmacogenomics journal. 2015;15:219-25.

4. Boye K, Jacob H, Frikstad KA, et al. Prognostic significance of S100A4 expression in stage II and III colorectal cancer: results from a population-based series and a randomized phase III study on adjuvant chemotherapy. Cancer medicine. 2016;5:1840-9.

5. Benson AB 3rd, Schrag D, Somerfield MR, et al. American Society of Clinical Oncology recommendations on adjuvant chemotherapy for stage II colon cancer. Journal of clinical oncology: official journal of the American Society of Clinical Oncology. 2004;22:3408-19.

6. Gao S, Tibiche C, Zou J, et al. Identification and Construction of Combinatory Cancer Hallmark-Based Gene Signature Sets to Predict Recurrence and Chemotherapy Benefit in Stage II Colorectal Cancer. JAMA oncology. 2016;2:37-45.

7. Fu J, Wu L, Fu W, et al How young is too young in breast cancer? -Young breast cancer is not a unique biological subtype. Clinical Breast Cancer. S1526820917300332.

8. Fu J, Wu L, Jiang M, et al Signet ring cell carcinoma of resectable metastatic colorectal cancer has rare surgical value. Journal of surgical oncology.

9. Benhaim L, Gerger A, Bohanes P, et al. Gender-specific profiling in SCN1A polymorphisms and time-torecurrence in patients with stage II/III colorectal cancer treated with adjuvant 5-fluoruracil chemotherapy. The pharmacogenomics journal. 2014;14:135-41.

10. Cho YK, Kim HC, Kim SH, et al. Location-related differences in sporadic microsatellite unstable colorectal cancer. Digestive liver disease: official journal of the Italian Society of Gastroenterology the Italian Association for the Study of the Liver. 2010;42:611-5.

11. Hugen $\mathrm{N}$, Verhoeven $\mathrm{RH}$, Lemmens $\mathrm{VE}$, et al. Colorectal signet-ring cell carcinoma: benefit from adjuvant chemotherapy but a poor prognostic factor. International journal of cancer. 2015;136:333-9. 
12. Chew MH, Yeo S-AE, Ng Z-P, et al Critical analysis of mucin and signet ring cell as prognostic factors in an Asian population of 2,764 sporadic colorectal cancers. International journal of colorectal disease; 25 : 1221-9.

13. Luo C, Cen S, Ding G, Wu W. Mucinous colorectal adenocarcinoma: clinical pathology and treatment options. Cancer Commun (Lond). 2019;39:13-.

14. Hugen N, Verhoeven RHA, Radema SA, et al. Prognosis and value of adjuvant chemotherapy in stage III mucinous colorectal carcinoma. Annals of oncology: official journal of the European Society for Medical Oncology. 2013;24:2819-24.

15. Allen WL, Dunne PD, McDade S, et al. Transcriptional subtyping and CD8 immunohistochemistry identifies poor prognosis stage II/III colorectal cancer patients who benefit from adjuvant chemotherapy. JCO precision oncology 2018; 2018.

16. Han Y, Lu S, Yu F, et al. A comparative analysis and guidance for individualized chemotherapy of stage II and III colorectal cancer patients based on pathological markers. Scientific reports. 2016;6:37240.

17. Cao B, Luo L, Feng L, et al. A network-based predictive gene-expression signature for adjuvant chemotherapy benefit in stage II colorectal cancer. Genes Chromosom Cancer. 2017;17:844.

18. Van Cutsem E, Cervantes A, Adam R, et al. ESMO consensus guidelines for the management of patients with metastatic colorectal cancer. Annals of oncology: official journal of the European Society for Medical Oncology. 2016;27:1386-422.

19. Lee YC, Su CY, Lin YF, et al. Lysosomal acid phosphatase 2 is an unfavorable prognostic factor but is associated with better survival in stage II colorectal cancer patients receiving chemotherapy. Oncotarget. 2017;8:12120-32.

20. Chang SY, Kuo CC, Wu CC, et al NKX6.1 hypermethylation predicts the outcome of stage II colorectal cancer patients undergoing chemotherapy. 2018; 57: 268-77.

21. Quah HM, Chou JF, Gonen M, et al. Identification of patients with high-risk stage II colon cancer for adjuvant therapy. Dis Colon Rectum. 2008;51:503-7.

22. Dienstmann R, Salazar R, Tabernero J. Personalizing colon cancer adjuvant therapy: selecting optimal treatments for individual patients. Journal of clinical oncology: official journal of the American Society of Clinical Oncology. 2015;33:1787-96.

23. Lin HH, Chang YY, Lin JK, et al. The role of adjuvant chemotherapy in stage II colorectal cancer patients. Int J Colorectal Dis. 2014;29:1237-43.

24. Kwon HY, Kim IK, Kang J, et al. In Vitro Adenosine Triphosphate-Based Chemotherapy Response Assay as a Predictor of Clinical Response to Fluorouracil-Based Adjuvant Chemotherapy in Stage II Colorectal Cancer. Cancer research treatment: official journal of Korean Cancer Association. 2016;48:970-7.

25. Deng $Y$, Wang $L$, Tan $S$, et al. KRAS as a predictor of poor prognosis and benefit from postoperative FOLFOX chemotherapy in patients with stage II and III colorectal cancer. Molecular oncology. 2015;9:1341-7.

26. Hutchins G, Southward K, Handley K, et al. Value of mismatch repair, KRAS, and BRAF mutations in predicting recurrence and benefits from chemotherapy in colorectal cancer. Journal of clinical oncology: official journal of the American Society of Clinical Oncology. 2011;29:1261-70. 
27. Huang $\mathrm{Y}, \mathrm{Yu} \mathrm{H}$, Lei $\mathrm{H}$, et al. Matrix metalloproteinase 7 is a useful marker for 5-fluorouracil-based adjuvant chemotherapy in stage II and stage III colorectal cancer patients. Med Oncol (Northwood Lond Engl). 2014;31:824.

28. Imaizumi H, Ishibashi K, Takenoshita S, Ishida H. Aquaporin 1 expression is associated with response to adjuvant chemotherapy in stage II and III colorectal cancer. Oncology letters. 2018;15:6450-6.

29. Feng Y, Li Y, Huang D, et al. HER2 as a potential biomarker guiding adjuvant chemotherapy in stage II colorectal cancer. European journal of surgical oncology: the journal of the European Society of Surgical Oncology the British Association of Surgical Oncology. 2019;45:167-73.

30. Dunne PD, McArt DG, O'Reilly PG, et al. Immune-Derived PD-L1 Gene Expression Defines a Subgroup of Stage II/III Colorectal Cancer Patients with Favorable Prognosis Who May Be Harmed by Adjuvant Chemotherapy. Cancer immunology research. 2016;4:582-91.

\section{Figures}

\section{Inclusion and exclusion criteria in SEER}

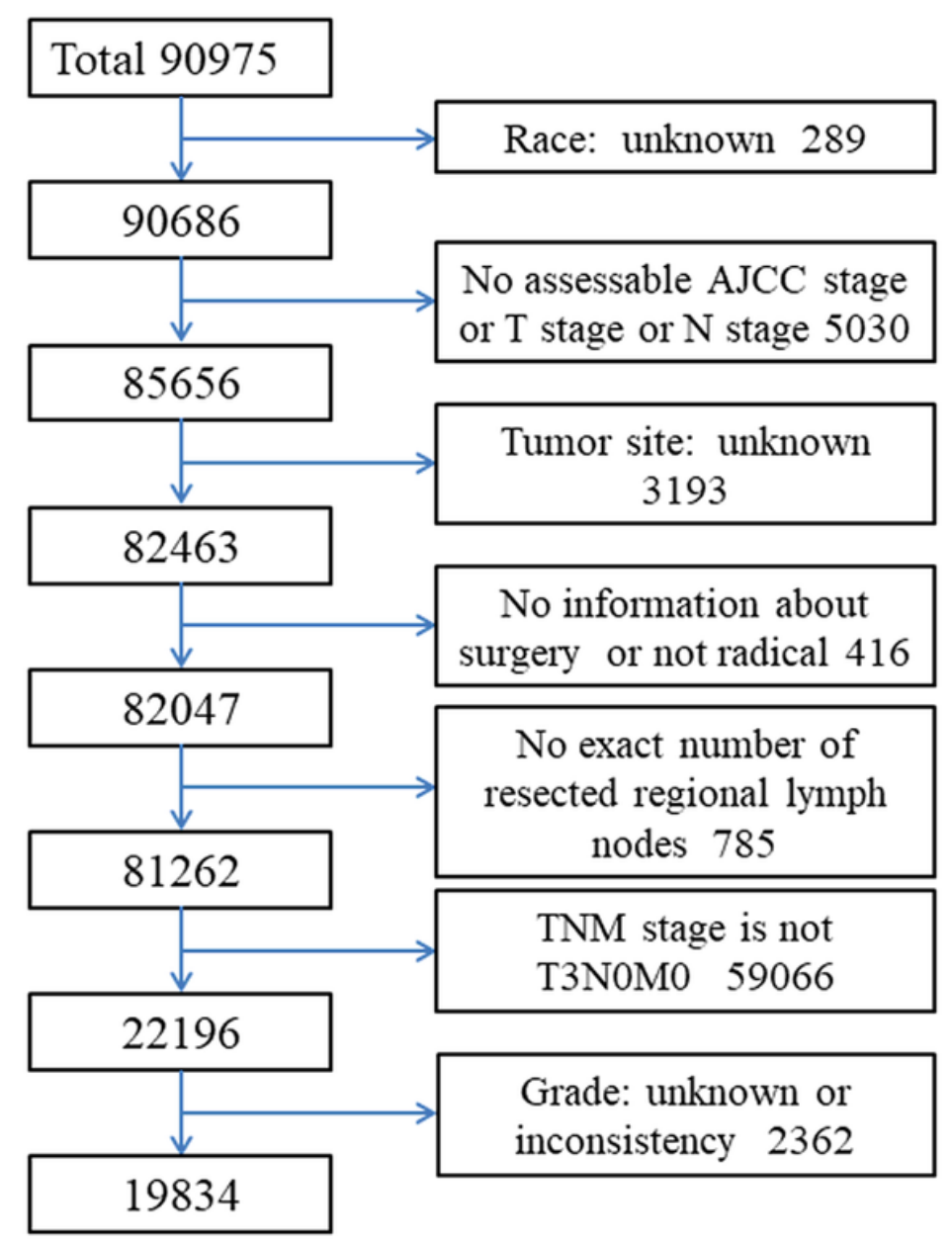

Selection in SEER

Sex $=$ male, female

Year of diagnosis $=2004-2012$

Site record $=$ colon excluding rectum, rectosigmoid junction

confirmation $=$ microscopically confirmed

Histology $=8140 / 3,8480 / 3,8490 / 3$

Survival months != unknown

Sequence $=$ one primary only

Age at diagnosis $=20-79$

Marital status $=$ single, married, divorced

Race recode=white, black, other

Grade=grade I, grade II, grade III, grade IV

Figure 1 
The inclusion and exclusion criteria in seer.

A

Cancer specific survival

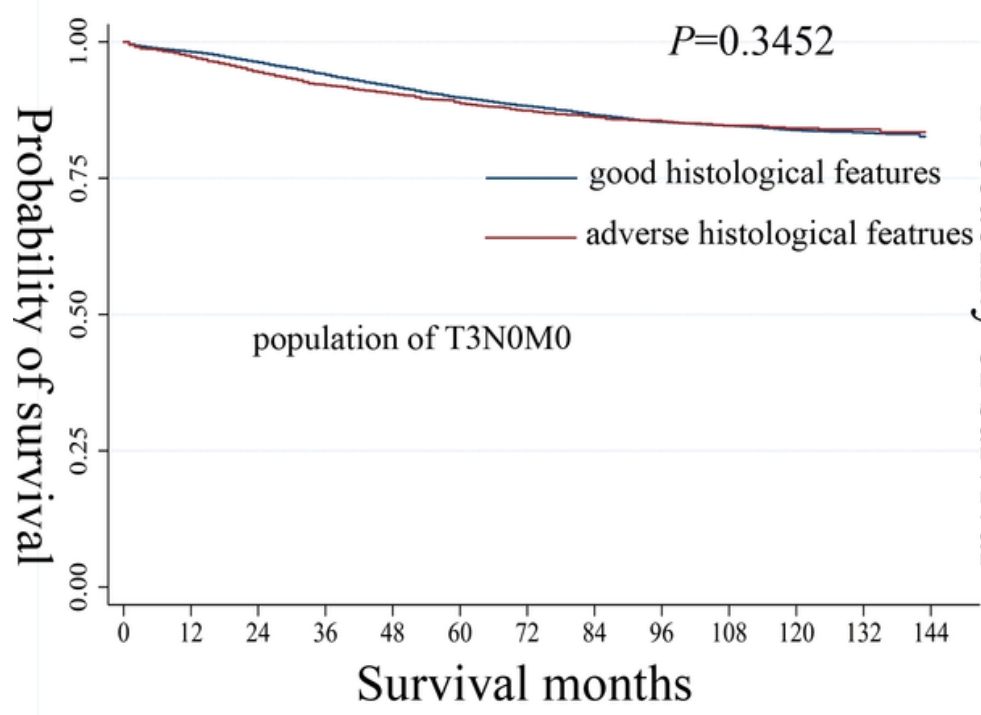

C

Cancer specific survival

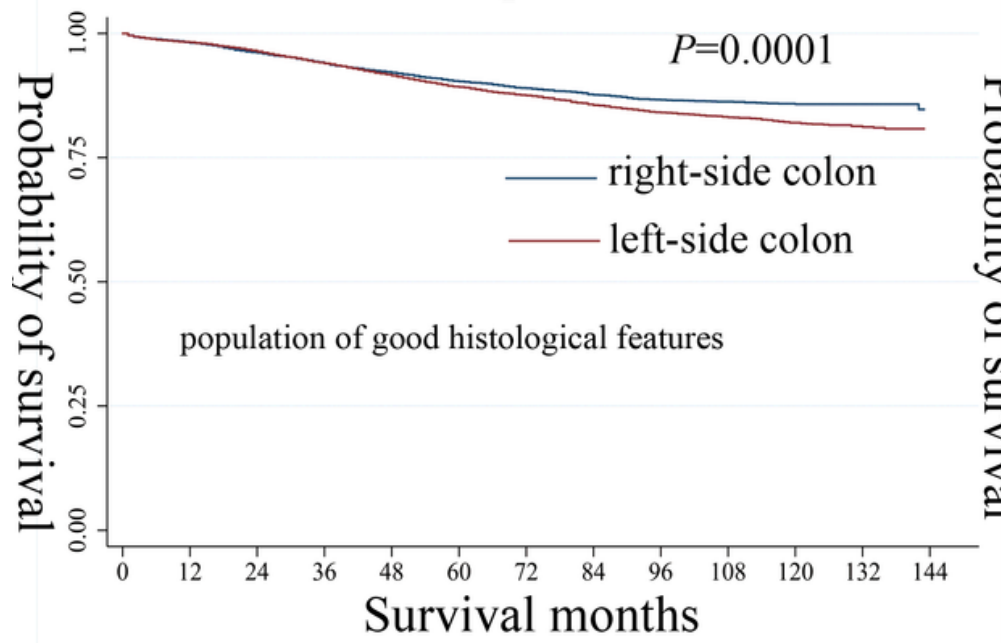

B Cancer specific survival

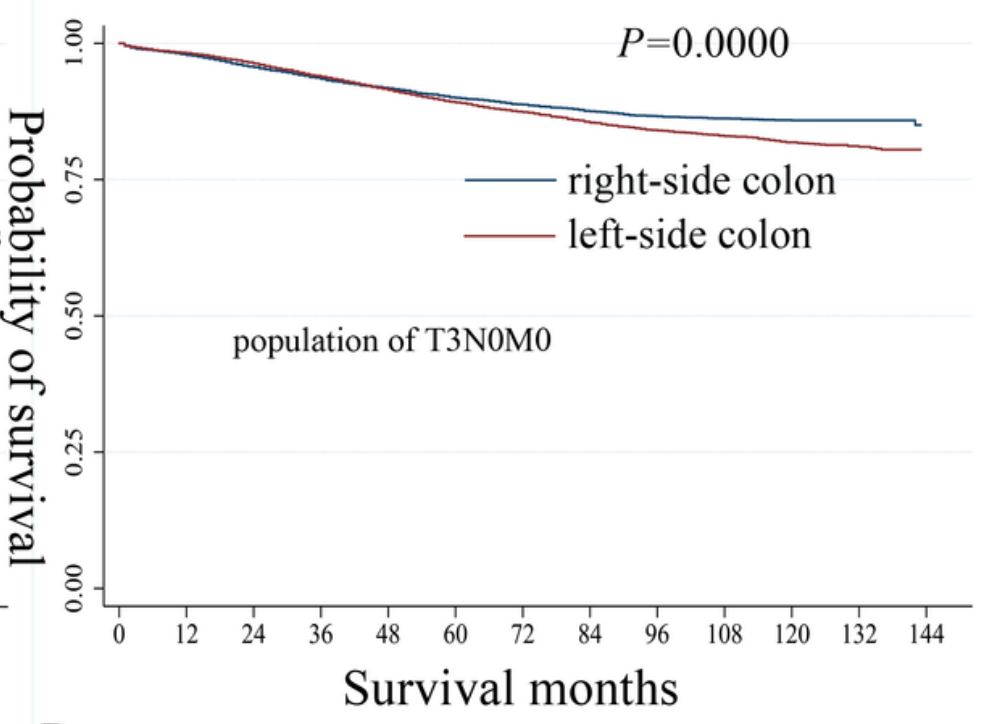

$\mathrm{D}$

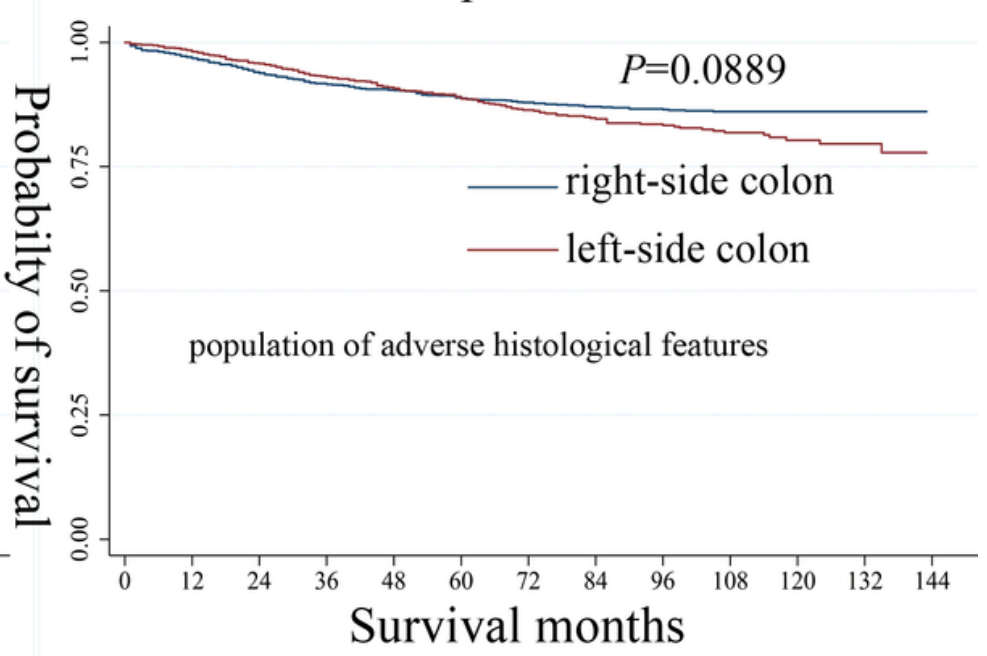

\section{Figure 2}

Survival analysis of colonic cancer patients with different features. A The cancer-specific survival (CSS) between good histological features (GD) and adverse histological features (BD) patients with T3N0M0 staging. B The CSS between right side and left side colon patients with T3NOMO staging. C The CSS between right side and left side colon patients of good histological features with T3N0M0 staging. D The CSS between right side and left side colon patients of adverse histological features with T3NOMO staging. P value from the log rank test. 

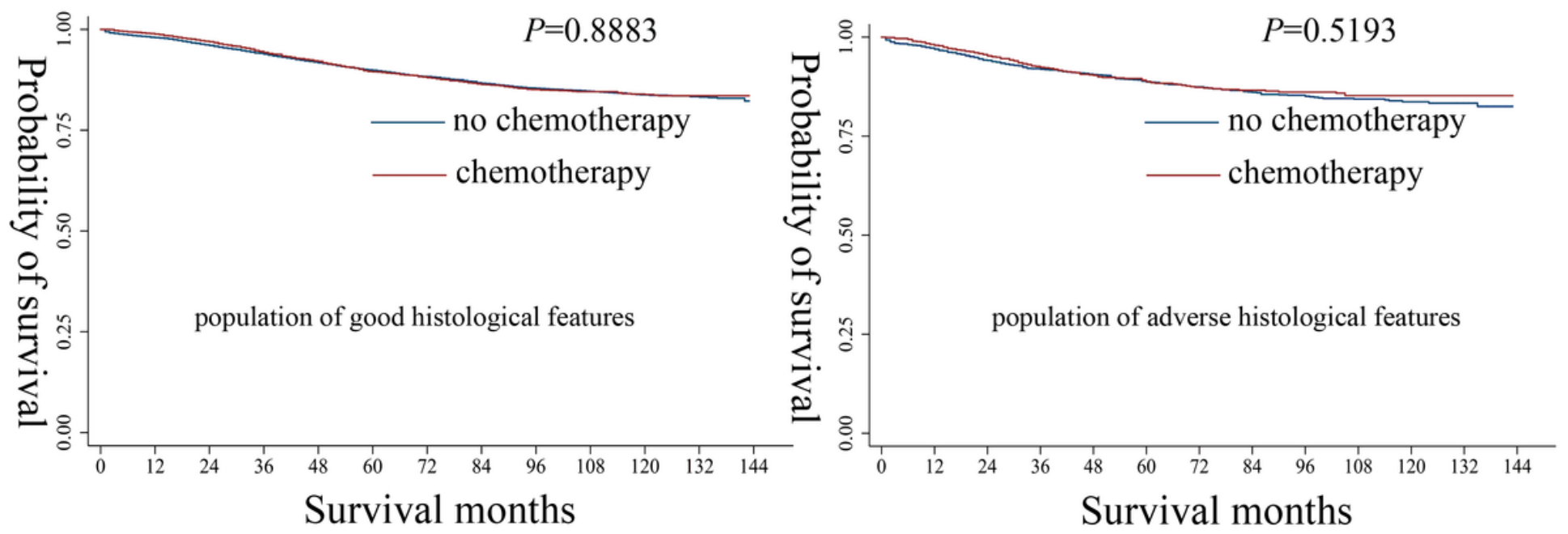

$\mathrm{C}$ Cancer specific survival

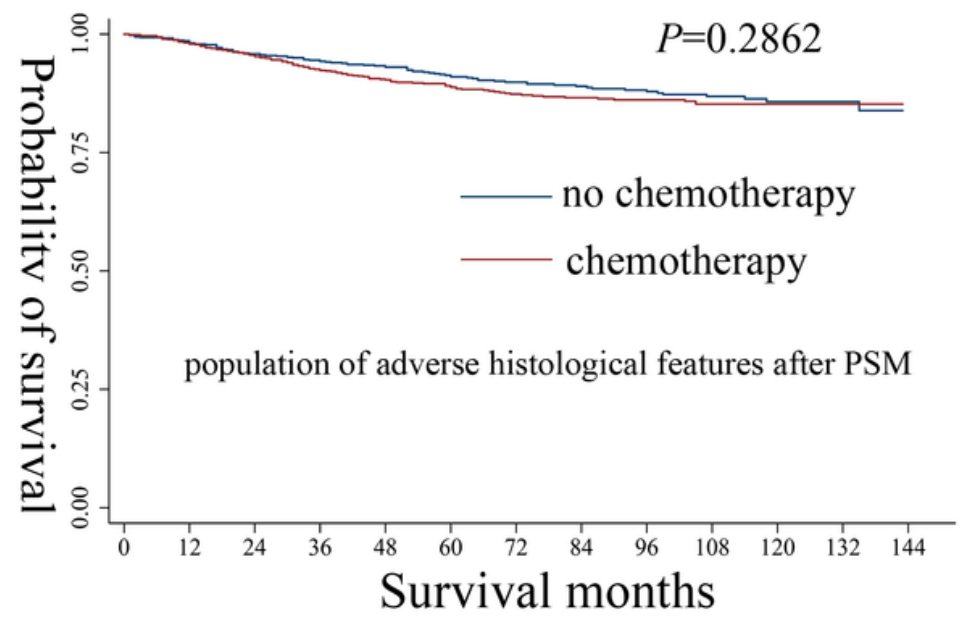

\section{Figure 3}

Survival analysis of adjuvant chemotherapy in colonic cancer patients with T3NOMO staging. A The cancerspecific survival (CSS) between patients of good histological features with T3NOMO staging with and without chemotherapy. B The CSS between patients of adverse histological features with T3NOMO staging with and without chemotherapy. C The CSS between patients of adverse histological features with T3NOMO staging with chemotherapy and without chemotherapy in PSM cohort. P value from the log rank test. 